\section{DRUG DELIVERY}

\section{Carbs deliver the goods}

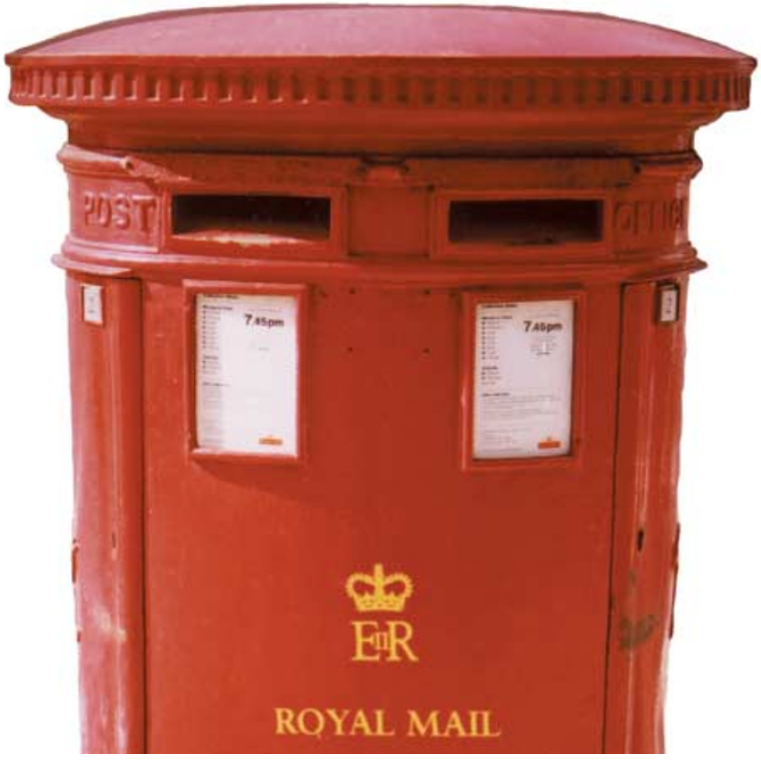

An approach to drug delivery using novel carbohydrates to carry drugs has recently been described in the Proceedings of the National Academy of Sciences. The method exploits the abundance of lectins, or carbohydrate-binding receptors, on the surface of specific cells. The authors demonstrate the delivery of the cancer drug doxorubicin targeted to hepatocytes in a mouse liver tumour model.

Known as the LEAPT strategy, lectin-directed enzyme-activated prodrug therapy is a two-stage delivery system. First, a novel nonmammalian glycosidase enzyme, rhamnosidase, is conjugated to a mammalian sugar; this is taken into the target cell by sugar-mediated receptor-mediated endocytosis, via the asialoglycoprotein receptor, a membrane-bound endocytic lectin found in abundance on the surface of hepatocytes in the liver. The second step involves administering the rhamnose-capped pro-drug; the drug is activated in the target cell once the sugar cap is removed by the pre-delivered rhamnosidase enzyme. The rhamnosidase enzyme was isolated from Penicillium decumbens and its carbohydrate structure was specifically engineered through enzymatic de-glycosylation and chemical re-glycosylation with mammalian sugar residues such as mannose and galactose.

Both in vitro and in vivo techniques proved the delivery of rhamnosidase and prodrug to hepatocytes and demonstrated successful activation of the rhamnose-bound prodrug. The authors tested the therapeutic effectiveness of LEAPT by constructing a doxorubicin prodrug, Rha-DOX, and using it in a hepatocellular carcinoma disease mouse model. After 42 days of therapy, the liver tumour burden was shown to have decreased by almost half compared with the control groups

\section{COMPUTATIONAL CHEMISTRY}

\section{Flexible approach to drug design}

A new model for structure-based drug design (SBDD) that better reflects the flexibility of proteins during ligand binding has been shown to successfully discriminate between known inhibitors and drug-like non-inhibitors of HIV protease (HIVp). The paper by Meagher and Carlson in the Journal of the American Chemical Society describes a rare example of successfully using an unliganded protein structure as the basis of SBDD.

In contrast to traditional SBDD, which uses a single $\mathrm{X}$-ray structure of a protein complexed with a ligand, this new model is based on multiple protein structures (MPS). A molecular dynamics computer simulation was used to provide conformational snapshots of the open, uncomplexed HIVp. A pharmacophore model of the HIVp active site (a threedimensional map of the required interactions between a ligand and HIVp) was created by aligning the protein snapshots, mapping the binding site with probe molecules, and clustering the probes to identify consensus regions that best represent complementary ligand functional groups - so-called pharmacophore elements. Because the model is based on an ensemble of protein conformations, it provides a more flexible model than one that is derived from a single conformation of the protein.

While optimizing the model, several parameters were varied, including the method of structural alignment, extent of conformational sampling (1-3 ns of snapshots), the size of the pharmacophore element and the number of pharmacophore elements that were required to constitute a 'hit'. The model was then evaluated for its ability to identify true inhibitors of HIVp over drug-like non-inhibitors of the enzyme.

The optimal model (created from 3 ns of snapshots and requiring 6 out of 6 pharmacophore elements) predicted $85 \%$ of known inhibitors as hits and only $11 \%$ of the non-inhibitors as false-positives. Significantly, the models that predicted hits with most accuracy were those that incorporated more protein flexibility by including snapshots over a greater period of time ( 2 and $3 \mathrm{~ns}$ ). Furthermore, when the authors attempted to develop a model based on a single structure (a so-called static model), they found that the number of sites of functionality shown by the probes was too complex to be of use in screening. By finding a consensus among the MPS, only the most important functional features were identified, which generated a more realistic and useful map of the pharmacophore elements.

Most remarkably, the model was able to predict the binding mode of known HIVp inhibitors, which is particularly impressive in light of the challenges posed by docking molecules to unliganded proteins and the large conformational change that occurs in HIVp on ligand binding.

The MPS method could therefore have a significant impact on SBDD by enabling chemists to begin designing drugs without first having to obtain a ligand-bound crystal structure of a drug target.

Joanna Owens

(2) References and links ORIGINAL RESEARCH PAPER Meagher, K. L. \& Carlson, H. A. Incorporating protein flexibility in structurebased drug discovery: using HIV-1 protease as a test case. J. Am. Chem. Soc. 126, 13276-13281 (2004) WEB SITE

Heather A. Carlson's Laboratory:

http://www.personal.umich.edu/ carlsonh/index.html 
receiving either Rha-DOX or glycosylated rhamnosidase alone.

LEAPT achieves higher concentrations of active drug at the target site through the use of engineered glycosylated enzymes than delivery of drug alone. Assuming that suitable receptors can be identified, delivery to other cell types is possible simply by changing the cognate sugar attached to the enzyme. The use of macrophage-associated carbohydrate receptors to treat macrophageassociated diseases such as lysosomal storage diseases or viral infections could benefit from this approach.

Melanie Brazil

(D) References and links ORIGINAL RESEARCh PAPER Robinson, M. A. et al. LEAPT: lectin-directed enzyme-activated prodrug therapy. Proc. Natl Acad. Sci. 24 Sep 2004 (doi:10.1073/pnas.0303574101) FURTHER READING

Duncan, R. The dawning era of polyme therapeutics. Nature Rev. Drug Discov. 2 347-360 (2003) | Prausnitz, M. R., Mitrogotri, S. \& Langer, R. Current status and future potential of transdermal drug delivery. Nature Rev. Drug Discov. 3, 115-124 (2004)

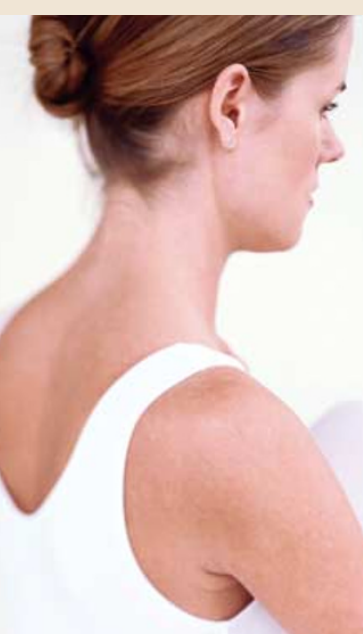

\section{Screen if you want to go faster!}

Historically, screening methods and diagnostics have been limited by the requirement to either directly label a chemical compound, biochemical molecule or cell using methods such as fluorescence or radioactivity; or to incorporate labelling stages, such as incubation with an antibody followed by a series of washes. These techniques, although sensitive and amenable to configuration in a high-throughput format, are expensive and there is the risk that altering the target molecule could affect its activity. Much effort has therefore been invested in developing label-free screening technologies, particularly as we enter an era in which faster methods for screening a glut of genomic targets are required. In the September issue of the Journal of Biomolecular Screening, four papers highlight novel label-free detection methods.

In the first paper, Huff et al. describe the use of the atomic force microscope (AFM) for the detection of proteins and pathogens, which can be coupled with protein nano-arrays. AFM uses a sharp, micron-scale tip to scan and amplify the topographical features of proteins and can therefore detect molecules bound to proteins by detecting nanometre changes in height. Coating the AFM tip with biological molecules provides the opportunity to bind specific proteins and can allow measurements of intermolecular binding constants, enabling the detection of single antibody-antigen interactions. The authors have developed a label-free, multiplexed, chip-based detection system that can directly determine the amount of virus particles in a sample.

In the second paper, Chambrone et al. describe how the measurement of bioimpedance can be used for the label-free detection and characterization of a cellular response to ligand-receptor binding. The method works by applying alternating voltages at set frequencies to cellular components (such as membranes) and intact cells, and monitors the resulting currents generated from the dipoles within the sample (reflected currents). The technique can detect different patterns of bioimpedance according to the particular receptor pathway being studied and the cell line being used, and could be applied in hit confirmation and pharmacological evaluation of receptor activity in response to an agonist or antagonist.

The third paper addresses one of the hardest challenges for screening technologies: detecting protein-protein interactions. The Biomolecular Interaction Detection (BIND) system described

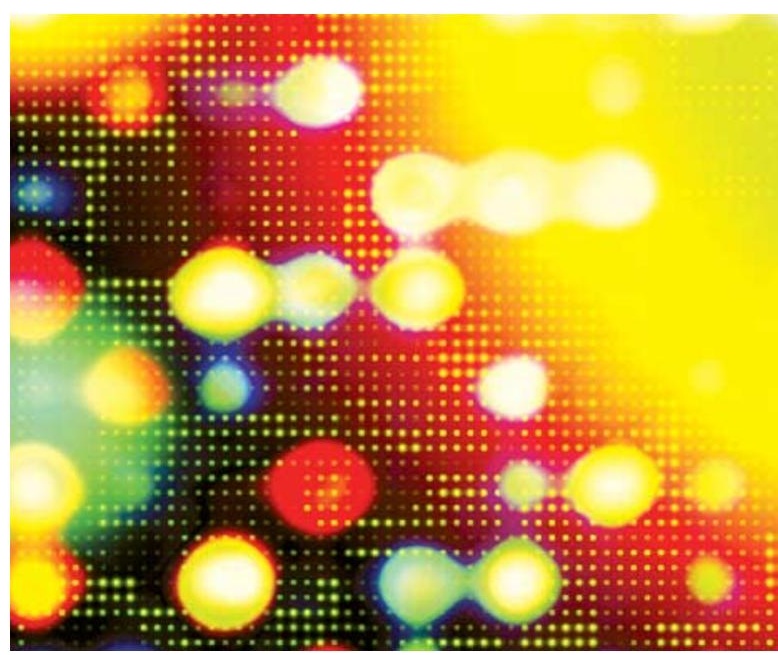

by Cunningham et al. is able to characterize these interactions, as well as small-molecule-protein interactions, protein-cell interactions and cell toxicity, in a high-throughput manner. BIND is a guided-mode resonant filter (GMRF) biosensor, which essentially means that on illumination with white light, the biosensor reflects only a narrow band of wavelengths. This narrow band can be optimized to provide a wavelength that is particularly sensitive to modulations that occur when a biochemical material is placed on its surface. The simple format of the BIND assay means that it can be manufactured as continuous sheets of plastic that can be tailored to suit 96-, 384- and 1,536-well assay formats.

A fourth paper by Zehender et al. describes a method for screening orphan or intractable targets, which is often hindered by stringent assay conditions that can miss low-affinity binders. The SpeedScreen comprises a homogenous solution-based affinity selection technique, followed by size-exclusion chromatography and microbore-liquid-chromatography/electrosprayionization mass spectrometry. The solutionbased affinity-selection method enables the detection of low-affinity binders that are often removed during the washing stages of methods in which the target is immobilized.

Joanna Owens

(0) References and links

ORIGINAL RESEARCH PAPERS Huff, J. L. et al. Label-free protein and pathogen detection using the atomic force microscope. J. Biomol. Screen. 9, 467-480 (2004) | Chambrone, G. J. et al. Cellular dielectric spectroscopy: a powerful new approach to label-free cellular analysis. J. Biomol. Screen. 9, 491-497 (2004) | Cunningham, B. T. et al. Labelfree assays on the BIND system. J. Biomol. Screen. 9, 481-490 (2004) Zehender, $\mathrm{H}$. et al. SpeedScreen: the 'missing link' between genomics and lead discovery. J. Biomol. Screen. 9, 498-505 (2004). 